\title{
The Relationship between Superhydrophobicity, Self-Cleaning Performance and Microstructure of Moth Wing
}

\author{
Gang Sun ${ }^{\mathrm{a}}$ and Yan Fang ${ }^{\mathrm{b}^{*}}$ \\ School of Life Science, Changchun Normal University, Changchun, Jilin, 130032, China \\ asungang@nenu.edu.cn, bangyan124@aliyun.com \\ * Corresponding author
}

\begin{abstract}
Keywords: Superhydrophobicity, Self-cleaning, Adhesion, Moth wing, Microstructure.
Abstract. The microstructure and superhydrophobicity of the moth wing surfaces were investigated by a scanning electron microscope (SEM), an atomic force microscope (AFM) and a contact angle (CA) meter. The relationship between superhydrophobicity, self-cleaning performance and microstructural characteristic was discussed. The wing surface is of low adhesion (sliding angle $1 \sim 4^{\circ}$ ) and high hydrophobicity $\left(\mathrm{CA} 151 \sim 158^{\circ}\right.$ ). The removal rate of $\mathrm{CaCO}_{3}$ pollution from the wing surface is as high as $86.7 \%$. There is a good positive correlation $\left(R^{2}=0.8883\right)$ between pollution removal rate and roughness index of the wing surface. The coupling effects of hydrophobic material and rough microstructure contribute to the complex wettability and remarkable self-cleaning property of the wing surface. Moth wing can be used as a template for design of micro-controllable superhydrophobic surface and nano self-cleaning material. This work may offer inspirations for preparation of novel interfacial material with multi-functions.
\end{abstract}

\section{Introduction}

Wettability is one of the important properties of solid surface, which is basically determined by chemical composition and microstructure of the surface. In the last few years, the interfacial materials with desirable properties and functions have attracted tremendous interest due to the wide applications in industrial, military, biomedical and domestic fields. The bio-surface is one of the most complicated three-dimensional periodical substrates and has become a popular bio-template because of its excellent characteristics [1]. In the previous work, the authors did some work on microstructure and wettability of butterfly wing [2]. In this work, the relationship between superhydrophobicity, self-cleaning performance and microstructure of the moth wing was investigated. The wetting mechanism was discussed from the perspective of biological coupling. The results may bring inspiration for design and preparation of smart interfacial materials.

\section{Materials and Methods}

Materials. The moth specimens of 10 species were collected in Changchun City, Jilin City and Dalian City. The wings were cleaned, desiccated and flattened, then cut into $5 \mathrm{~mm} \times 5 \mathrm{~mm}$ pieces from the discal cell (Fig. 1). The distilled water for measurements of CA and sliding angle (SA) was purchased from Tianjin Pharmaceuticals Group Co. Ltd., China. The volume of water droplets was 5 $\mu \mathrm{l}$. The size distribution of $\mathrm{CaCO}_{3}$ particle was $5 \sim 10 \mu \mathrm{m}$.
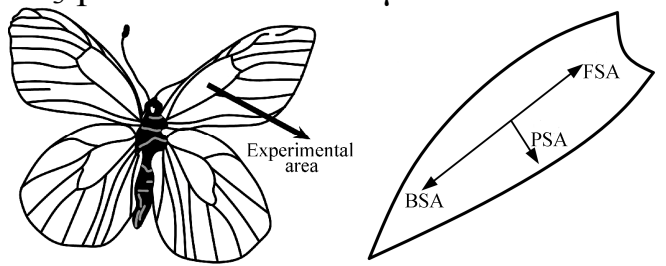

Fig. 1 The experimental area and the sliding angles in different directions on the wing surface

Characterization of Microstructure. After gold coating by an ion sputter coater (Hitachi E-1045, Japan), the wing pieces were observed and photographed by a SEM (Hitachi SU8010, Japan) and an 
AFM (Bruker Dimension Icon, USA). Using Photoshop software, the microstructural parameters of the butterfly wing surfaces were measured in SEM images.

Measurements of Wetting Angles. Using an optical CA measuring system (DataPhysics OCA20, Germany), the CA of water droplets on the wing surfaces was measured via sessile drop method at ambient conditions of $(25 \pm 1){ }^{\circ} \mathrm{C}$ and relative humidity of approximately $80 \%$. The SA of water droplets was measured along three different directions, including forward SA (FSA, the SA of droplets from wing base to wing terminal end), backward SA (BSA, the SA of droplets from wing terminal end to wing base), and perpendicular SA (PSA, the SA of droplets perpendicular to the major axis of wing) (Fig. 1). The water droplets were dripped on the sample table in a horizontal position, then the inclination degree of the table was raised $1^{\circ}$ each time until the droplets rolled off freely. The inclination degree of the table was recorded as the SA value.

Removal of $\mathrm{CaCO}_{3}$ Particles. The wing pieces were affixed to glass slides with double-sided adhesive tape, and put on the sample table of OCA20. Five mg of $\mathrm{CaCO}_{3}$ particles were evenly spread on the discal cell of the wing. A water droplet from an injector fell on the $\mathrm{CaCO}_{3}$ area. The sample table was inclined $3^{\circ}$, and the droplet flowed through the contaminated area. A stereo microscope (Zeiss SteREO Discovery V12, Germany) was used to observe the removal of $\mathrm{CaCO}_{3}$ particles. An electronic analytical balance (Shimadzu AUX-120, Japan) was used to measure the mass of $\mathrm{CaCO}_{3}$ residual on the wing surface. The removal rate was calculated.

\section{Results and Discussion}

Micro-morphology of the Wing Surfaces. The wing surfaces display complicated micro/nano structures. The micrometric scales constitute the primary structure [Fig. 2(a)]. The length of the scales is $58 \sim 135 \mu \mathrm{m}$, the width is $32 \sim 82 \mu \mathrm{m}$, the spacing is $55 \sim 126 \mu \mathrm{m}$ (Table 1). The submicrometric longitudinal ridges and lateral bridges on the scales constitute the secondary structure [Fig. 2(b)]. The height of the longitudinal ridges is $0.23 \sim 0.65 \mu \mathrm{m}$, the width is $0.27 \sim 0.92 \mu \mathrm{m}$, the spacing is $0.88 \sim 1.69$ $\mu \mathrm{m}$ (Table 1). The nano stripes on the longitudinal ridges and lateral bridges constitute the tertiary structure [Fig. 2(b)]. The cross-section of the longitudinal ridge is triangular [Fig. 2(c)].

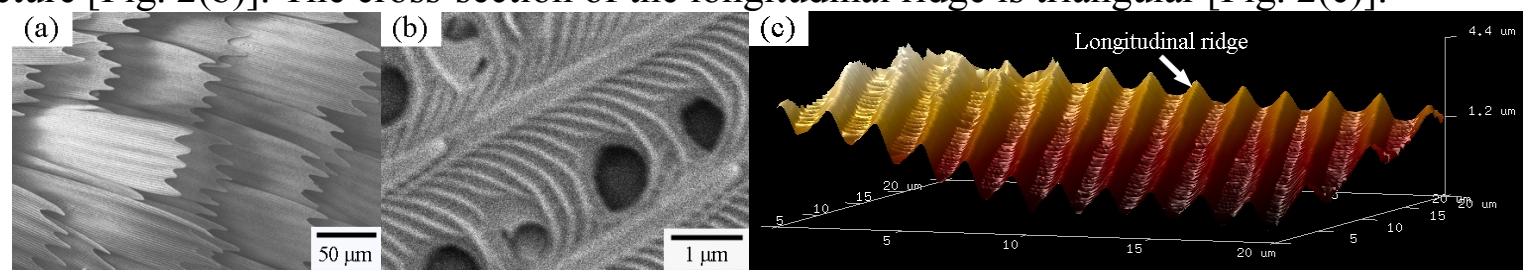

Fig. 2 The micro/nano structures of the moth wing surfaces

(a) Primary structure (SEM); (b) Secondary structure and tertiary structure (SEM); (c)

Three-dimensional image of the wing surface (AFM).

Table 1 Microstructural parameters and wettability of the moth wing surfaces

\begin{tabular}{|c|c|c|c|c|c|c|c|c|c|c|c|}
\hline \multirow{2}{*}{ Butterfly species } & \multicolumn{3}{|c|}{ Scale $(\mu \mathrm{m})$} & \multicolumn{3}{|c|}{ Longitudinal ridge $(\mu \mathrm{m})$} & \multicolumn{2}{|c|}{$\mathrm{CA}\left(^{\circ}\right)$} & \multicolumn{3}{|c|}{ Measured SA $\left({ }^{\circ}\right)$} \\
\hline & Length $(a)$ & Width $(b)$ & Spacing $(c)$ & Height $(d)$ & Width $(e)$ & Spacing $(f)$ & Measured CA & Predicted CA & FSA & BSA & PSA \\
\hline$\overline{\text { Angerona prunaria }}$ & 63 & 32 & 55 & 0.41 & 0.35 & 1.41 & 153 & 154 & 3 & 9 & 7 \\
\hline Calothysanis comptaria & 102 & 48 & 66 & 0.23 & 0.81 & 1.55 & 152 & 154 & 2 & 11 & 5 \\
\hline Celyphoides cespitanus & 94 & 39 & 57 & 0.38 & 0.44 & 1.05 & 157 & 158 & 4 & 17 & 8 \\
\hline Epagoge groticna & 135 & 62 & 85 & 0.49 & 0.27 & 1.48 & 158 & 160 & 2 & 8 & 10 \\
\hline Holcocerus vicarious & 108 & 78 & 126 & 0.47 & 0.48 & 1.69 & 152 & 153 & 1 & 11 & 9 \\
\hline Naxa seriaria & 71 & 43 & 82 & 0.65 & 0.33 & 1.26 & 156 & 156 & 3 & 6 & 12 \\
\hline Parasa sinica & 58 & 58 & 95 & 0.52 & 0.53 & 1.37 & 151 & 154 & 4 & 12 & 9 \\
\hline Plutella xylostella & 70 & 47 & 62 & 0.64 & 0.92 & 0.88 & 154 & 155 & 1 & 6 & 14 \\
\hline Selenia sordidaria & 86 & 65 & 84 & 0.44 & 0.37 & 0.95 & 153 & 152 & 3 & 9 & 11 \\
\hline Tineola translucens & 69 & 82 & 69 & 0.35 & 0.39 & 1.27 & 156 & 157 & 2 & 8 & 8 \\
\hline Average & 86 & 55 & 78 & 0.46 & 0.49 & 1.29 & 154 & 155 & 3 & 10 & 9 \\
\hline
\end{tabular}

Low Adhesive Superhydrophobicity of the Wing Surfaces. The moth wing surfaces are highly hydrophobic $\left(\mathrm{CA} 151 \sim 158^{\circ}\right.$ ), the average value is $154^{\circ}$. Meanwhile, the water SAs are extremely small (FSA $1 \sim 4^{\circ}$, BSA $6 \sim 17^{\circ}$, PSA $5 \sim 14^{\circ}$ ) (Table 1 ). The complex wettability of the butterfly wing surface resembles that of the lotus leaf [3] and the rice leaf [4] with low adhesion and high hydrophobicity, but differs from the peanut leaf [5] and the rose petal [6] with high adhesive 
superhydrophobicity. There are significant differences between the SAs in various directions $(p<0.01)$, FSA $<$ PSA $<$ BSA (Table 1). The asymmetrical sliding behavior of water droplet on the wing surface results from the anisotropic micro/nano structures and the different energy barriers. Even a very slight tilting $\left(1 \sim 4^{\circ}\right)$ of the wing is sufficient to cause the droplet to roll off readily and take away the contaminating particles efficiently.

The Relationship between Superhydrophobicity and Microstructure of the Wing Surface. The butterfly wing surface is relatively rough with superhydrophobicity and heterogeneity. A composite contact is formed between the droplet and the surface. Thus, the contact behavior can be expressed by the Cassie-Baxter equation:

$$
\cos \theta_{c}=\phi_{s} \cos \theta_{e}+\phi_{s}-1
$$

where $\theta_{c}$ is the apparent CA of a droplet on a heterogeneous composite surface, $\emptyset_{s}$ is the area fraction of solid on a composite surface $\left(0<\emptyset_{S}<1\right.$, calculated from microstructural parameters of the wing surface), $\theta_{e}$ is the intrinsic CA of water on an ideal flat surface (approximately $95^{\circ}$ on the moth wing). The contact state of a water droplet on the micro/nano structure of the wing surface is shown in Fig. 3.

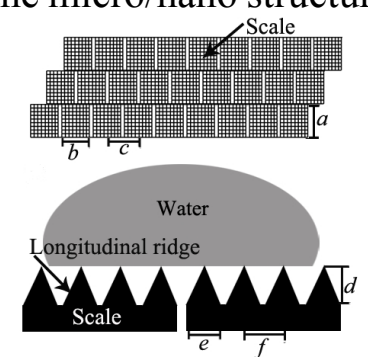

Fig. 3 The contact state of water on the micro/nano structure of the wing surfaces

In this case, Eq. (1) can be modified for the theoretical (predicted) CA $\left(\theta_{t}\right)$ as follows:

$$
\cos \theta_{t}=\sqrt{\frac{4 d^{2}}{e^{2}}+1} * \frac{b e}{c f} * \cos \theta_{e}+\frac{b e}{c f}-1
$$

Based on Eq. (2), the predicted CAs were calculated (Table 1). Taking predicted CAs as independent variable $y^{*}$, measured CAs as dependent variable $y$, the degree of fitting was judged by:

$$
\begin{aligned}
& Q=\sum\left(y-y^{*}\right)^{2} \\
& R_{\text {New }}=1-\left(Q / \sum y^{2}\right)^{1 / 2}
\end{aligned}
$$

where $Q$ is sum of square of deviations, $R_{\mathrm{New}}$ is the coefficient of determination in nonlinear regression equation. The calculated $R_{\mathrm{New}}$ values are $0.934 \sim 0.968$ for the 10 moth species. There is no significant difference between the measured CAs and the predicted CAs, demonstrating the microstructural model for hydrophobicity is in good accord with the Cassie equation.

In the light of Eq. (2), the smaller the values of $\sqrt{\frac{4 d^{2}}{e^{2}}+1}$ and $\frac{b e}{c f}$ are, the bigger the theoretical CAs are. Namely, smaller width and bigger spacing of the micrometric scale, as well as smaller height, smaller width and bigger spacing of the nano longitudinal ridge can lead to stronger hydrophobicity of the wing surfaces. Naturally hydrophobic material such as chitin is the chemical foundation for the high hydrophobicity on the moth wing surfaces. Much higher hydrophobicity, however, cannot be induced by the chemical composition alone. Superhydrophobicity of the wing surfaces is attributed to a combination of hydrophobic material and rough micro/nano structure.

The Relationship between Self-Cleaning Performance and Microstructure of the Wing Surface. The wing surface exhibits remarkable self-cleaning performance. When a droplet flows over the wing surface, most $\mathrm{CaCO}_{3}$ particles are taken away. The average removal rate is $86.7 \%$ (Fig. 4). The removal rate has no significant correlation $\left(R^{2}<0.1\right)$ with the scale parameters (length, width, spacing) or the longitudinal ridge parameters (height, width, spacing), but has a significant correlation $\left(R^{2}=0.8883\right)$ with roughness index $(R I)$ of the wing surface (Fig. 4). RI, the magnitude of surface roughness, is the ratio of the real area to the geometry projection area. The rough micro-texture on the wing surface plays a crucial role in the complex wettability. In a contrast test, the scales were removed 
from the wing surfaces. The CA values reduce by $18.4 \sim 46.1^{\circ}$. All the SAs (FSA, BSA, PSA) increase above $65^{\circ}$ (the maximum inclination angle of the sample table is $65^{\circ}$ ).

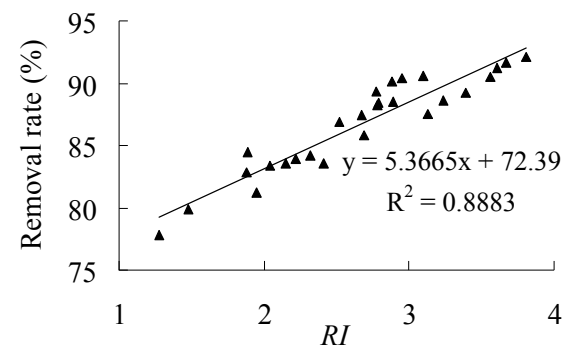

Fig. 4 The relationship between the removal rate of $\mathrm{CaCO}_{3}$ particle and roughness index $(R I)$ of the wing surface

\section{Summary}

The moth wing surface is of low adhesion (water SA $1 \sim 4^{\circ}$ ) and superhydrophobicity (water CA $151 \sim 158^{\circ}$ ). In micro-dimension, the smaller the width or the bigger the spacing of the scale is, the stronger the hydrophobicity of the wing surfaces is. In nano-dimension, the smaller the height or the smaller the width or the bigger the spacing of the longitudinal ridge is, the stronger the hydrophobicity of the wing surfaces is. The average rate of $\mathrm{CaCO}_{3}$ particle removal from the wing surface is $86.7 \%$. The wing surface possesses hierarchical micro/nano structures. There is a good positive correlation $\left(R^{2}=0.8883\right)$ between $\mathrm{CaCO}_{3}$ removal rate (self-cleaning performance) and roughness index of the wing surface. The coupling effect of hydrophobic material and rough microstructure contributes to the special complex wettability and excellent self-cleaning performance of the wing. This work may bring inspirations for preparation of micro-controllable superhydrophobic surface, self-cleaning nano material and smart interfacial material.

\section{Acknowledgements}

This work was financially supported by the Natural Science Foundation of Jilin Province, China (201115162), Science and Technology Project of Educational Department of Jilin Province, China (2012357, 2011186). Dr. Prof. Yan Fang is the corresponding author of this paper.

\section{References}

[1] G. Sun, Y. Fang, Q. Cong, Anisotropism of the non-smooth surface on butterfly wing, J. Bionic Eng. 6 (2009) 71-76.

[2] Y. Fang, G. Sun, Y.H. Bi, H. Zhi, Multiple-dimensional micro/nano structural models for hydrophobicity of butterfly wing surfaces and coupling mechanism, Science Bulletin 60 (2015) 256-263.

[3] X. Yao, Y.L. Song, L. Jiang, Applications of bio-inspired special wettable surfaces, Adv. Mater. 23 (2011) 719-734.

[4] L. Feng, S.H. Li, Y.S. Li, H.J. Li, L.J. Zhang, J. Zhai, Y.L. Song, B.Q. Liu, L. Jiang, D.B. Zhu, Super-hydrophobic surfaces: from natural to artificial, Adv. Mater. 14 (2002) 1857-1860.

[5] B. Bhushan, E.K. Her, Fabrication of superhydrophobic surfaces with high and low adhesion inspired from rose petal, Langmuir 26 (2010) 8207-8217.

[6] M.H. Jin, X.J. Feng, L. Feng, Superhydrophobic aligned polystyrene nanotube films with high adhesive force, Adv. Mater. 17 (2005) 1977-1981. 